\title{
BENTHONIC FORAMINIFERA OF THE ANTARCTIC OCEAN
}

$\operatorname{AUTHOR}(\mathrm{S})$ :

Uchio, Takayasu

CITATION:

Uchio, Takayasu. BENTHONIC FORAMINIFERA OF THE ANTARCTIC

OCEAN. SPECIAL PUBLICATIONS FROM THE SETO MARINE BIOLOGICAL LABORATORY 1960, 1(12): 1-20

\section{ISSUE DATE:}

1960-05

URL:

http://hdl.handle.net/2433/176442

RIGHT: 
SPECIAL PUBLICATIONS FROM THE SETO MARINE BIOLOGICAL LABORATORY

BIOLOGICAL RESULTS

$\mathrm{OF}$

THE JAPANESE ANTARCTIC RESEARCH EXPEDITION

12.

\title{
BENTHONIC FORAMINIFERA OF THE ANTARCTIC OCEAN
}

\author{
BY \\ TAKAYASU UCHIO \\ PETROLEUM ENGINEERING INSTITUTE \\ UNIVERSITY OF TOKYO \\ BUNKYO.KU, TOKYO, JAPAN
}

SIRAHAMA, WAKAYAMA-KEN

JAPAN

MAY 1960 
SPECIAL PUBLICATIONS FROM THE SETO MARINE BIOLOGICAL LABORATORY

\author{
BIOLOGICAL RESULTS \\ $\mathrm{OF}$
}

THE JAPANESE ANTARCTIC RESEARCH EXPEDITION

12.

\title{
BENTHONIC FORAMINIFERA OF \\ THE ANTARCTIC OCEAN
}

BY

TAKAYASU UCHIO

PETROLEUM ENGINEERING INSTITUTE

UNIVERSITY OF TOKYO

BUNKYO-KU, TOKYO, JAPAN

SIRAHAMA, WAKAYAMA-KEN

JAPAN

MAY 1960 
THIS SERIES contains THE BIOLOGICAL RESULTS OF THE JAPANESE ANTARCTIC RESEARCH EXPEDITION and is published by the Seto Marine Biological Laboratory. Parts will appear at irregular intervals as they become ready.

PRINTED IN JAPAN

BY NIPPON PRINTING AND PUBLISHING Co., Ltd.

HULUSTMA, OSAKA 


\section{INTRODUCTION}

THE eleven sediment samples here studied were collected by Dr. Daitaro SHoJI aboard the "SÔYA" during the Second Japanese Antarctic Research Expedition, 1957-58. Results on the grain size distributions and the taxonomic work on the Radiolaria in these samples were published by ShoJI \& Sato (1959), and by NAKASEKO (1959), respectively. The planktonic Foraminifera in these samples, as well as in two plankton-net samples, have been analyzed by the present author and the results appeared in a separate paper (UCHi, 1960). A preliminary report (UCHIO, 1959) on the Foraminifera was presented at the 66th annual meeting of the Geological Society of Japan held in April, 1959, in Tokyo. A paper on bottom deposits, collected by the members of the Tokyo University of Fisheries aboard the "UMITAKA-MARU" in 1957 from the same area, was published by NIINo (1958). The writer wishes to thank Dr. Takasi Tokioka of the Kyoto University, Dr. Fred B PHLEGER and Miss Frances L. PARKER of the Scripps Institution of Oceanography of the University of California for their constant encouragement and for reading the manuscript.

\section{LOCATIONS OF THE SAMPLES}

The locations of these sediment samples are shown in Figure 1 and are listed in Table 1. The bathymetry (Fig. 1) is based on a chart published by the International Hydrographic Bureau, with additional sounding data obtained by the scientists aboard the "UMTTAKA-MARU" (1957) and "SÔYA" (1958).

Bottom sampling was done with a small dredge at all stations except Station 8, where a modified PHLEGER bottom sampler (a short gravity corer) was used. All samples were dried on the deck of the survey ship "SOYA", and a part of the sample at Station 2 was preserved in formalin.

The sediment at all stations is sand except at three where mud is found. The boundary of the two sediment types lies at a depth of approximately $850 \mathrm{~m}$. These sediments contain various kinds, sizes and amounts of subangular to angular ice-rafted glacial material.

\section{BENTHONIC FORAMINIFERA ASSEMBLAGES}

\section{(I) Biococioses}

A part of the sediment sample at Station 2 was preserved in formalin. The rose Bengal staining technique (WALTON, 1952) was used to distinguish the 
living Foraminifera from the empty tests. The following species were identified as living when collected:

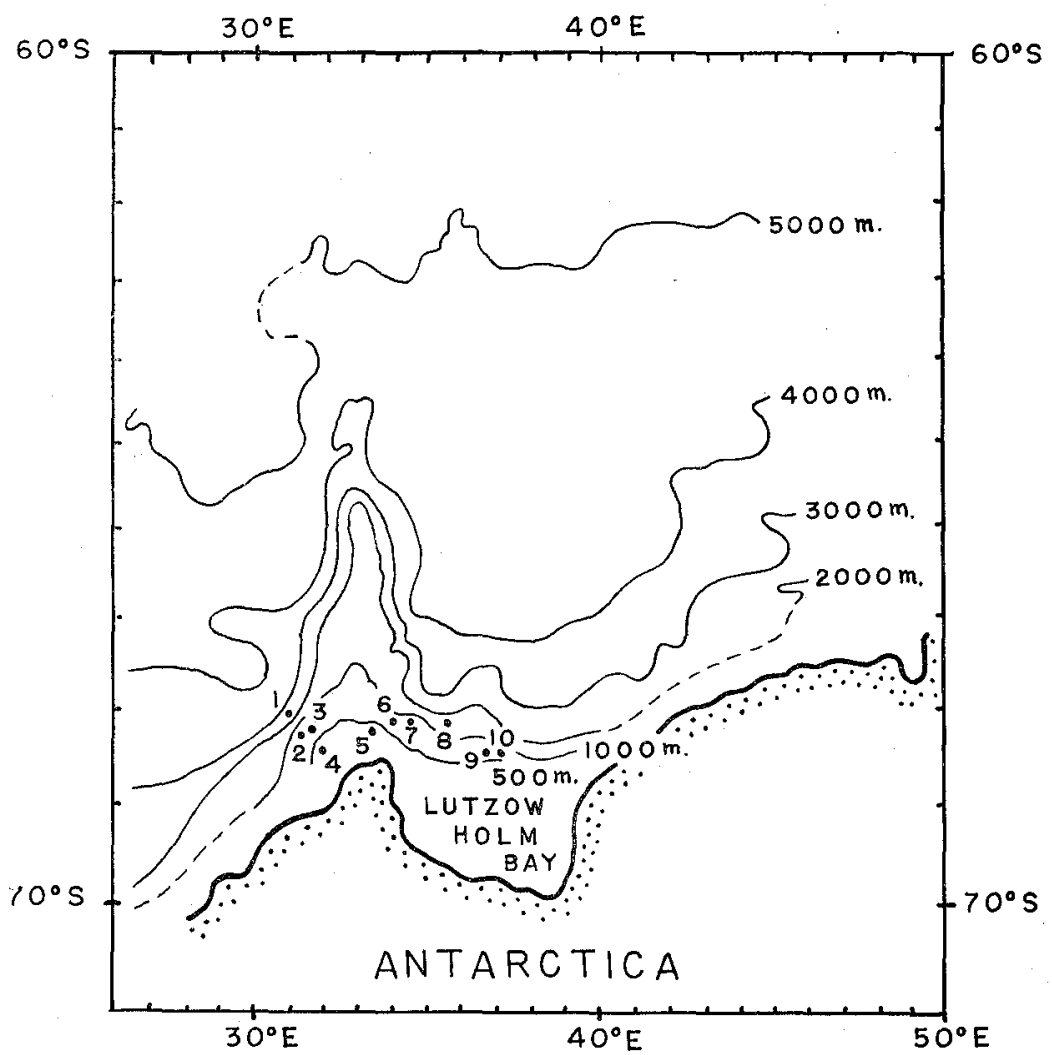

Fig. 1. Locations of stations and submarine topography.

Table 1. Locations of stations, depths, dates of collection and sediment types.

\begin{tabular}{|c|c|c|c|c|c|}
\hline Station & S. Lat. & E. Long. & Depth & Date of collection & Sediment type \\
\hline 1 & $68^{\circ} 10.3^{\prime}$ & $31^{\circ} \quad 12.5^{\prime}$ & $2480 \mathrm{~m}$ & Feb. 5, 1958 & "Muds" \\
\hline 2 & $68^{\circ} 19.2^{\prime}$ & $31^{\circ} \quad 21.2^{\prime}$ & $700 \mathrm{~m}$ & Feb. 2,1958 & medium Sands \\
\hline 3 & $68^{\circ} \quad 17.4^{\prime}$ & $31^{\circ} \quad 46.9^{\prime}$ & $700 \mathrm{~m}$ & Feb. 1,1958 & medium Sands \\
\hline $3^{\prime}$ & $68^{\circ} \quad 17.4^{\prime}$ & $31^{\circ} \quad 46.9^{\prime}$ & $590 \mathrm{~m}$ & Feb. 1,1958 & coarse Sands \\
\hline 4 & $68^{\circ} \quad 29.7^{\prime}$ & $32^{\circ} \quad 02.7^{\prime}$ & $350 \mathrm{~m}$ & Jan. 31,1958 & Sands (poorly sorted) \\
\hline 5 & $68^{\circ} 14.7^{\prime}$ & $33^{\circ} \quad 37.0^{\prime}$ & $495 \mathrm{~m}$ & Jan. 28,1958 & medium Sands \\
\hline 6 & $68^{\circ} 10.0^{\prime}$ & $34^{\circ} 04.0^{\prime}$ & $620 \mathrm{~m}$ & Jan. 27, 1958 & coarse Sands \\
\hline 7 & $68^{\circ} \quad 09.7^{\prime}$ & $34^{\circ} \quad 34.0^{\prime}$ & $830 \mathrm{~m}$ & Jan. 26, 1958 & coarse Sands, Shells \\
\hline 8 & $68^{\circ} 06.0^{\prime}$ & $\begin{array}{ll}35^{\circ} \quad 08.0^{\prime} \\
\end{array}$ & $1900 \mathrm{~m}$ & Jan. 24, 1958 & "Muds" \\
\hline 9 & $68^{\circ} 32.0^{\prime}$ & $36^{\circ} \quad 30.0^{\prime}$ & $870 \mathrm{~m}$ & Feb. 9, 1958 & "Muds", Gravels \\
\hline 10 & $68^{\circ} 30.9^{\prime}$ & $\begin{array}{ll}37^{\circ} & 08.7^{\prime}\end{array}$ & $560 \mathrm{~m}$ & Feb. 8,1958 & Sands, "Muds" \\
\hline
\end{tabular}




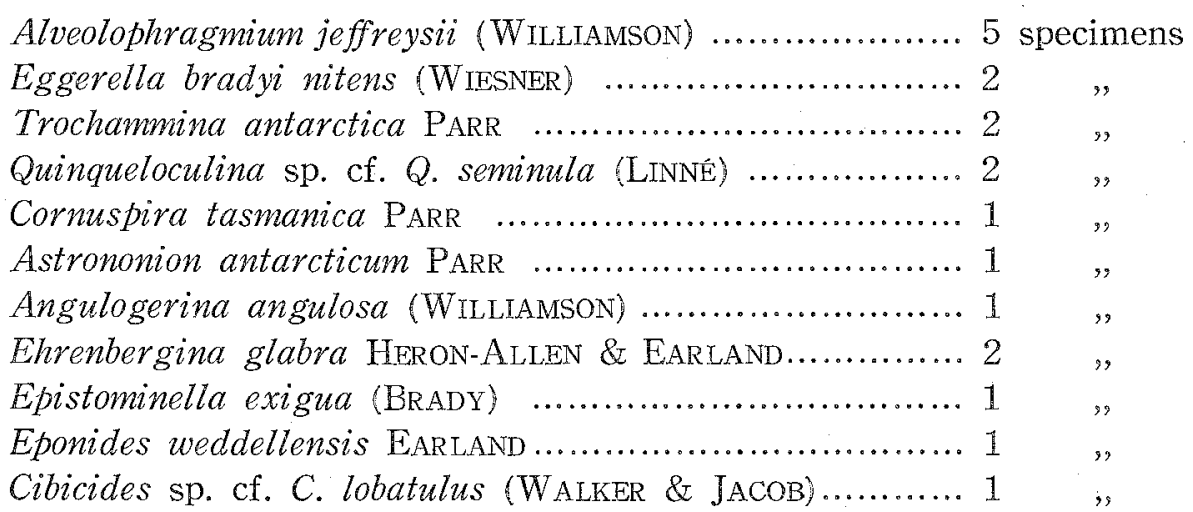

This sample was not collected for quantitative analysis but it may suggest the relative abundances of these species.

\section{(II) Thanatocoenoses}

In the preliminary report (UCHIO, op. cit.) the writer examined Foraminifera fractions of relatively larger sizes and not of the smaller sizes. In the present report Foraminifera of all fractions are analyzed quantitatively, and slightly modified results are obtained. The average number of benthonic Foraminifera counted at each station is ca. 550, except at Station 8 where only 9 specimens are found in all the material available 7.1 grams). Frequency distributions (in \%) of the species at each station are listed in Table 2. Three faunal assemblages are distinguished, with depth boundaries at approximately $850 \mathrm{~m}$ and $2,000 \mathrm{~m}$. There must be at least one additional assemblage shallower than these three. Unfortunately the bottom sediments shallower than $350 \mathrm{~m}$ cannot be collected because the shallow bottom close to Antarctica is always covered by a thick ice cap.

These assemblages characteristically consist of two groups of species: (1) indigenous species around Antarctica (PEARCEY 1914; HERON-AlLEN \& EARLAND 1922, 1932; WIESNER 1931; EARland 1933, 1934, 1936; ChAPMAN \& Parr 1937 ; PARr 1950), (2) cosmopolitan species in the Arctic waters (NorVANG 1945; Cushman 1948; PhLEgER 1952; LoEBLICH \& TAPPAN 1953) and in the cold, deep waters of the Atlantic, Pacific and perhaps Indian Oceans (BRADY 1884; FLINT 1899 ; Cusiman 1910-1917, 1918-1931; Höglund 1947 ; Phleger 1951 ; Phleger, PARKER \& PEIRSON 1953; PARKER 1954\%. The absence of Eggerella advena (Cushman); Buliminella elegantissima (D'ORBIGNY), Buccella frigida (CUSHMAN), species of Elphidium and Elphidiella in the present material, though they are known in the Antarctic and Arctic waters, is to be expected considering the depths from which the material was obtained. The absence of Uvigerina in this material as well as in other parts of the Antarctic region is remarkable, though the genus is known in the Subantarctic region (HERON-ALLEN \& 
Takayasu Úchı

Table 2. Occurrences of benthonic Foraminifera in percent of total (living plus dead) population. ( $\mathrm{R}=$ rare, $\mathrm{vR}=$ very rare)

\begin{tabular}{|c|c|c|c|c|c|c|c|c|c|c|c|}
\hline Station & 4 & 5 & 10 & $3^{\prime}$ & 6 & 3 & 2 & 7 & 9 & 8 & \\
\hline Depth is & 350 & 495 & 560 & 590 & 620 & 700 & 700 & 830 & 870 & 1900 & 02 \\
\hline Total population/1gr. & 332 & 350 & 2345 & 2656 & 6842 & 8416 & 1335 & 65 & 2891 & & 47 \\
\hline$\%$ & 34 & 97 & 75 & 70 & 96 & 97 & 97 & 92 & 63 & 96 & 8 \\
\hline$\%$ of & 66 & 3 & 25 & 30 & 4 & 3 & 3 & 8 & 37 & 4 & 1 \\
\hline Total benth & 220 & 372 & 587 & 792 & 250 & 255 & 548 & & 1077 & 1 & 80 \\
\hline A in $\%$ & 19 & 5 & 14 & 9 & 1 & 2 & 1 & 33 & 9 & 22 & \\
\hline 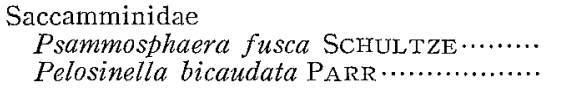 & $\overline{\mathrm{R}}$ & $\begin{array}{l}\mathrm{vR} \\
\mathrm{vR}\end{array}$ & $\overline{\mathrm{R}}$ & - & $\begin{array}{l}\mathrm{vR} \\
--\end{array}$ & $\begin{array}{l}\mathrm{vR} \\
\mathrm{vR}\end{array}$ & $\begin{array}{l}\mathrm{vR} \\
\mathrm{vR}\end{array}$ & $\stackrel{33}{-}$ & $\overline{\mathrm{R}}$ & - & $\overline{\mathrm{vR}}$ \\
\hline 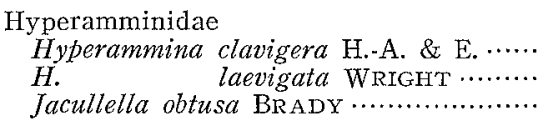 & $\overline{-}$ & $\begin{array}{l}-\overline{v R} \\
\mathrm{vR}\end{array}$ & $\begin{array}{l}\mathrm{vR} \\
\mathrm{VR} \\
\mathrm{VR}\end{array}$ & E & $\begin{array}{l}- \\
-\end{array}$ & $\begin{array}{l}- \\
-\end{array}$ & $\begin{array}{l}-\bar{v} \\
\mathrm{vR} \\
\mathrm{vR}\end{array}$ & - & $\begin{array}{l}- \\
\mathrm{vR} \\
\mathrm{R}\end{array}$ & - & - \\
\hline 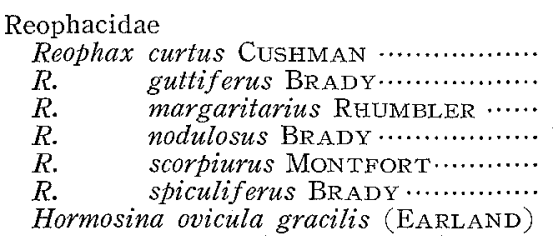 & $\begin{array}{l}- \\
- \\
\mathrm{vR} \\
\overline{\mathrm{vR}} \\
- \\
-\end{array}$ & $\begin{array}{l}\overline{-} \\
\overline{-} \\
\overline{\mathrm{R}} \\
\overline{-}\end{array}$ & $\begin{array}{l}\mathrm{vR} \\
\mathrm{VR} \\
\mathrm{VR} \\
\mathrm{VR} \\
\mathrm{VR} \\
\mathrm{vR}\end{array}$ & $\begin{array}{l}- \\
E \\
E \\
- \\
-\end{array}$ & $\begin{array}{l}- \\
- \\
- \\
- \\
-\end{array}$ & $\begin{array}{l}- \\
- \\
- \\
- \\
-\end{array}$ & $\begin{array}{l}- \\
- \\
- \\
\text { vR } \\
- \\
-\end{array}$ & $\begin{array}{l}- \\
- \\
- \\
- \\
-\end{array}$ & $\begin{array}{l}\overline{v R} \\
\overline{v R} \\
\overline{v R} \\
R \\
\text { vR }\end{array}$ & $\begin{array}{l}- \\
- \\
- \\
- \\
-\end{array}$ & $\begin{array}{l}- \\
- \\
\overline{\mathrm{vR}} \\
- \\
- \\
-\end{array}$ \\
\hline 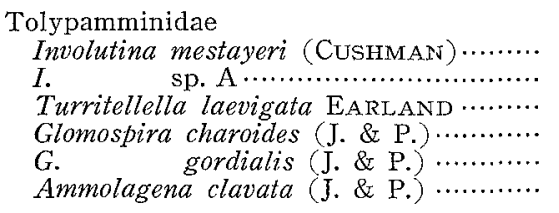 & $\begin{array}{l}\overline{\mathrm{R}} \\
\overline{-} \\
\overline{-}\end{array}$ & $\begin{array}{l}- \\
\mathrm{vR} \\
\mathrm{vR} \\
\mathrm{vR} \\
\overline{\mathrm{R}}\end{array}$ & $\begin{array}{l}\overline{\mathrm{vR}} \\
\overline{\mathrm{R}} \\
\mathrm{vR}\end{array}$ & $\begin{array}{l}- \\
- \\
- \\
- \\
- \\
-\end{array}$ & $\begin{array}{l}- \\
\frac{-}{\mathrm{R}} \\
\mathrm{R}\end{array}$ & $\begin{array}{l}- \\
- \\
- \\
2\end{array}$ & $\begin{array}{l}\mathrm{vR} \\
- \\
- \\
\bar{R}\end{array}$ & $\begin{array}{l}\overline{\mathrm{vR}} \\
\overline{-} \\
- \\
-\end{array}$ & $\begin{array}{l}- \\
- \\
\text { R } \\
-\end{array}$ & $\begin{array}{l}- \\
- \\
- \\
- \\
-\end{array}$ & $\frac{\overrightarrow{\mathrm{vR}}}{\overline{\mathrm{R}}}$ \\
\hline 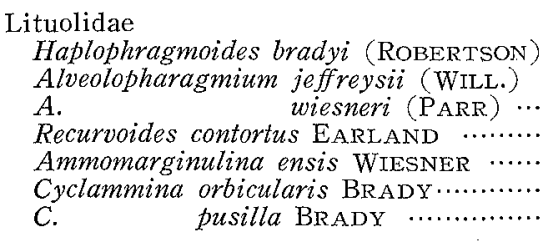 & $\begin{array}{l}- \\
R \\
R \\
\mathrm{VR} \\
- \\
-\end{array}$ & $\begin{array}{l}\frac{-}{2} \\
\frac{\mathrm{vR}}{-} \\
-\end{array}$ & $\begin{array}{l}\mathrm{R} \\
\mathrm{R} \\
\mathrm{VR} \\
\overline{\mathrm{VR}} \\
\mathrm{vR}\end{array}$ & $\begin{array}{l}\bar{R} \\
\mathrm{R} \\
\overline{\mathrm{R}} \\
\overline{-} \\
-\end{array}$ & $\begin{array}{l}\overline{\mathrm{R}} \\
\overline{-} \\
- \\
-\end{array}$ & $\begin{array}{l}\overline{\mathrm{vR}} \\
\overline{-} \\
\overline{-} \\
\overline{-}\end{array}$ & $\begin{array}{l}\overline{\mathrm{R}} \\
\overline{\mathrm{vR}} \\
\mathrm{vR} \\
\mathrm{vR}\end{array}$ & $\begin{array}{l}\overline{\mathrm{vR}} \\
\overline{-} \\
\overline{-} \\
\overline{-}\end{array}$ & $\begin{array}{l}\overrightarrow{\mathrm{R}} \\
\mathrm{R} \\
\mathrm{R} \\
\mathrm{R} \\
\mathrm{vR}\end{array}$ & $\begin{array}{l}\overline{-} \\
\overline{11} \\
- \\
-\end{array}$ & $\begin{array}{l}\mathrm{vR} \\
- \\
\mathrm{VR} \\
\mathrm{R} \\
-\end{array}$ \\
\hline 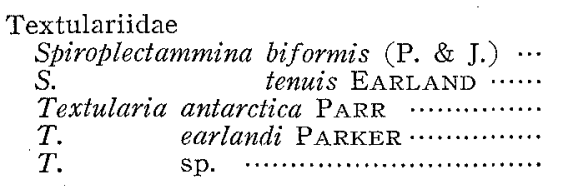 & $\begin{array}{l}- \\
1 \\
2 \\
-\end{array}$ & $\begin{array}{l}\mathrm{vR} \\
\mathrm{vR} \\
\mathrm{vR}\end{array}$ & $\begin{array}{l}- \\
\overline{\mathrm{R}} \\
1 \\
\end{array}$ & $\begin{array}{l}- \\
\overline{2} \\
-\end{array}$ & $\begin{array}{l}- \\
\overline{\mathrm{vR}} \\
-\end{array}$ & $\begin{array}{l}- \\
\bar{E} \\
-\end{array}$ & $\begin{array}{l}- \\
\overline{\mathrm{L}} \\
\overline{-}\end{array}$ & $\begin{array}{l}- \\
\overline{-} \\
-\end{array}$ & $\begin{array}{l}\frac{\mathrm{R}}{\mathrm{R}} \\
\frac{1}{1}\end{array}$ & $\begin{array}{l}- \\
- \\
-\end{array}$ & $\begin{array}{l}\mathrm{v} \\
\mathrm{v}\end{array}$ \\
\hline 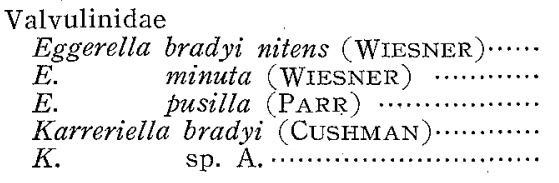 & $\begin{array}{c}\mathrm{vR} \\
1 \\
\mathrm{R} \\
- \\
-\end{array}$ & $\begin{array}{l}\mathrm{R} \\
\mathrm{VR} \\
- \\
-\end{array}$ & $\begin{array}{l}\mathrm{R} \\
\mathrm{R} \\
\mathrm{R} \\
\mathrm{VR} \\
-\end{array}$ & $\begin{array}{l}\mathrm{R} \\
- \\
- \\
-\end{array}$ & $\begin{array}{l}\mathrm{vR} \\
\frac{-}{-} \\
-\end{array}$ & $\begin{array}{l}\mathrm{vR} \\
- \\
- \\
-\end{array}$ & $\begin{array}{l}\mathrm{R} \\
- \\
-\end{array}$ & $\begin{array}{l}\overline{-} \\
\overline{\mathrm{vR}} \\
\overline{\mathrm{vR}}\end{array}$ & $\begin{array}{l}\mathrm{R} \\
\mathrm{R} \\
\mathrm{R} \\
-\end{array}$ & $\begin{array}{l}11 \\
- \\
- \\
-\end{array}$ & $\mathrm{R}$ \\
\hline $\begin{array}{ll}\text { Botellina (?) goesii EARLAND } & \text { (?............. } \\
B . & \text { (?) tasmanica PARR } \ldots \ldots \ldots \ldots\end{array}$ & - & $\overrightarrow{\mathrm{vR}}$ & $\begin{array}{l}\mathrm{vR} \\
\mathrm{vR}\end{array}$ & E & $\underline{\mathrm{vR}}$ & - & - & - & - & - & - \\
\hline
\end{tabular}


Benthonic Foraminifera of the Antarctic Ocean

Table $\underset{x}{2}$ (Continued)

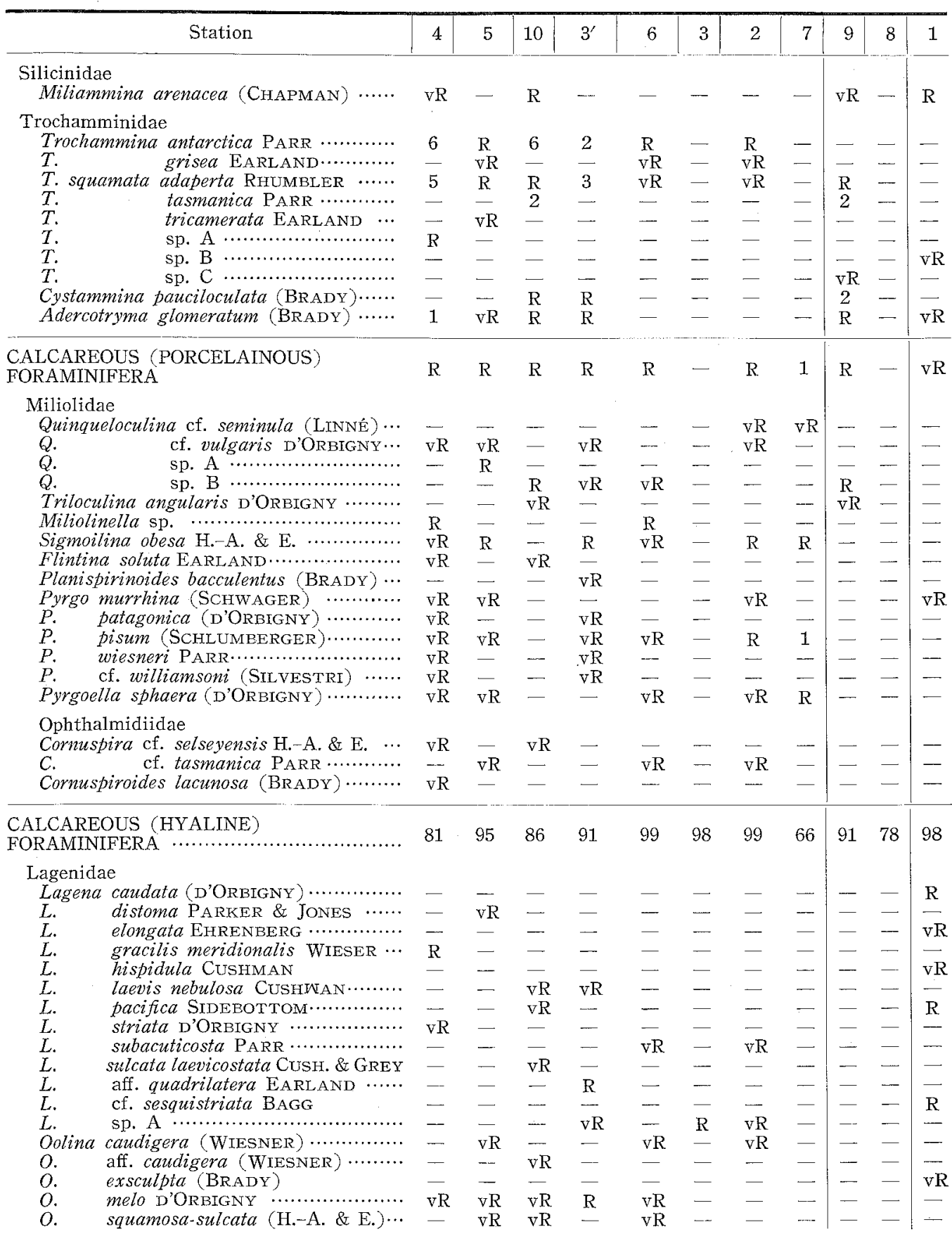


Takayasu Uchio

2

Table $\mathcal{X}$ (Continued)

\begin{tabular}{|c|c|c|c|c|c|c|c|c|c|c|c|}
\hline Station & 4 & 5 & 10 & $3^{\prime}$ & 6 & 3 & 2 & 7 & 9 & 8 & 1 \\
\hline sterigera (BRADY) var. …........... & - & $\mathrm{vR}$ & - & - & - & $\mathrm{R}$ & - & - & $\mathrm{vR}$ & - & \\
\hline$\ldots \ldots \ldots \ldots \ldots+\cdots$ & - & $\mathrm{vF}$ & 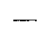 & & $\mathrm{vR}$ & & $\mathrm{R}$ & $\mathrm{vR}$ & - & - & - \\
\hline cf. globosa & - & - & - & - & 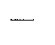 & 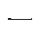 & & 4 & - & - & - \\
\hline$O . \quad$ sp. A $\cdots \ldots . .$. & - & $\mathrm{vR}$ & 一 & - & 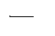 & - & - & - & - & - & - \\
\hline Fissurina alveolata & - & $\mathrm{vR}$ & - & - & - & - & - & - & - & - & - \\
\hline cellulata (WIESNER) …….......... & - & & & - & - & & - & - & $\mathrm{vR}$ & - & - \\
\hline 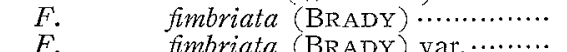 & $\mathrm{vR}$ & 1 & $\mathrm{vR}$ & $\mathrm{R}$ & $\mathrm{R}$ & $\mathrm{R}$ & 2 & - & - & - & - \\
\hline 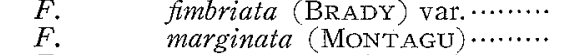 & $\mathrm{R}$ & - & - & $\mathrm{R}$ & & & $\mathrm{R}$ & - & $\mathrm{R}$ & & \\
\hline (............. & $\underline{-}$ & $\mathrm{vR}$ & $\mathrm{R}$ & $\underline{\mathrm{K}}$ & $\begin{array}{l}\mathrm{R} \\
\mathrm{VR}\end{array}$ & - & K. & - & $\mathrm{K}$ & - & - \\
\hline R) $\cdots$ & - & - & - & - & $\mathrm{vR}$ & - & - & - & - & - & \\
\hline subfo & $\mathrm{vR}$ & - & $\mathrm{vR}$ & - & - & $\ldots$ & - & - & - & & - \\
\hline N) $\cdots$ & - & - & $\mathrm{vR}$ & $v R$ & - & - & - & - & - & - & - \\
\hline$\cdots \cdots$ & - & - & & - & - & - & -- & - & - & - & $\mathrm{vR}$ \\
\hline$F$. & $\mathrm{vR}$ & -. & $\mathrm{vR}$ & - & - & - & - & - & - & - & - \\
\hline$F . \quad$ sp. A $\cdots$ & - & - & & - & - & & - & & $\mathrm{R}$ & - & - \\
\hline Parafissurina abno & - & $\mathrm{vR}$ & $\mathrm{vR}$ & - & - & - & $\mathrm{R}$ & & & - & - \\
\hline ........... & - & $\mathrm{vR}$ & & - & - & - & - & $\mathrm{vR}$ & - & - & - \\
\hline$P$. & - & - & $\mathrm{vR}$ & - & - & - & - & - & - & -1 & $-\bar{P}$ \\
\hline fusuliform & - & - & & - & - & - & - & $-\mathrm{P}$ & $\mathrm{vR}$ & - & $v R$ \\
\hline …...... & - & $\mathrm{vR}$ & & $\overline{-}$ & - & - & - & $\mathrm{vR}$ & - & - & \\
\hline ...... & - & $\mathrm{vR}$ & $\mathrm{vR}$ & $\mathrm{R}$ & $\mathrm{R}$ & - & $\mathrm{R}$ & & - & -1 & - \\
\hline ...... & - & & - & $\mathrm{R}$ & & 4 & - & - & - & - & - \\
\hline ......... & - & $\mathrm{vR}$ & $\mathrm{R}$ & $\mathrm{R}$ & - & - & - & - & - & 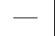 & - \\
\hline$P . \quad$ sp. & - & 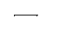 & & & & - & - & $\mathrm{P}$ & $\mathrm{R}$ & - & \\
\hline Dentalina cf. $c$ & - & - & & - & vR & - & - & $\mathrm{vR}$ & $\overline{\mathrm{n}}$ & - & \\
\hline comn & - & $\mathrm{vR}$ & & & & & - & - & $\mathrm{R}$ & - & $\mathrm{R}$ \\
\hline ... & $\mathrm{vR}$ & - & $\mathrm{vl}$ & - & $\mathrm{vR}$ & - & - & - & & - & \\
\hline$\cdots$ & & - & & - & & - & - & - & $\mathrm{vR}$ & - & \\
\hline ....... & - & $\mathrm{vR}$ & - & - & - & - & - & - & - & - & \\
\hline ....... & - & - & - & - & $\mathrm{vR}$ & $\mathrm{vR}$ & $\mathrm{R}$ & - & - & -1 & $\mathrm{vR}$ \\
\hline .......... & - & - & 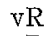 & - & & - & - & - & - & - & \\
\hline ....... & - & - & $v$ & - & - & - & 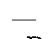 & - & - & 一 & \\
\hline inas & - & - & & $\mathrm{vH}$ & - & vR & $\mathrm{vR}$ & - & - & - & - \\
\hline ....... & - & - & - & - & - & - & & - & - & & \\
\hline Ast & - & - & & - & - & - & - & - & $\mathrm{R}$ & - & $\ldots$ \\
\hline Mar & - & - & 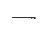 & - & - & - & & & $\mathrm{R}$ & - & \\
\hline Marginul & - & - & & - & - & - & $\mathrm{vR}$ & - & & - & \\
\hline Robulus angt & - & - & $\mathrm{vR}$ & - & - & - & - & - & $\mathrm{R}$ & - & \\
\hline asteri & - & $\mathrm{vR}$ & & $\mathrm{R}$ & -- & - & $\ldots$ & -.. & & - & \\
\hline & - & - & - & - & - & - & - & & - & - & - \\
\hline$R$ & - & - & - & - & - & - & & & - & - & \\
\hline sp. C & - & - & - & - & $\mathrm{vR}$ & - & $\mathrm{vR}$ & - & - & - & - \\
\hline Darbyella sp. & $\mathrm{vR}$ & & - & - & - & - & & $\mathrm{vR}$ & & 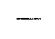 & \\
\hline sp. B & - & - & - & - & - & - & $\mathrm{vR}$ & & -- & - & - \\
\hline \multicolumn{12}{|l|}{ Polymorpl } \\
\hline$G$ & - & $\mathrm{vR}$ & - & 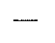 & - & - & - & - & - & - & \\
\hline a extensa (CusH & - & - & - & & & - & - & - & & & \\
\hline …........... & $\overline{\mathrm{vR}}$ & 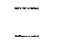 & $\overline{\mathrm{vR}}$ & - & - & 二 & - & $\overline{\mathrm{vR}}$ & $\ldots$ & - & \\
\hline & 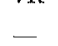 & - & - & 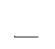 & $\mathrm{vR}$ & - & - & - & - & $\ldots$ & \\
\hline sp. $\cdots$ & - & $\mathrm{vR}$ & - & $\ldots$ & - & - & $\ldots$ & - & - & - & \\
\hline \multicolumn{12}{|l|}{ Nonion } \\
\hline ( & $\mathrm{R}$ & $\mathrm{vl}$ & $\mathrm{R}$ & $\mathrm{R}$ & $\mathrm{R}$ & & $\mathrm{R}$ & - & & - & \\
\hline & $\mathrm{R}$ & & $\mathrm{R}$ & $x^{2}+2$ & $\mathrm{R}$ & & $\mathrm{R}$ & - & $\mathrm{R}$ & - & \\
\hline & 1 & - & $I^{\prime}$ & & & $\mathrm{vR}$ & & - & $\mathrm{R}$ & & \\
\hline & $\mathrm{R}$ & $\cdots$ & & $\mathrm{vR}$ & - & - & $\cdots$ & - & $\mathrm{R}$ & $\overline{-}$ & \\
\hline Nonionella scapha bradyi (CHAPMAN & $\mathrm{K}$ & & & & & & & & & & \\
\hline
\end{tabular}


Benthonic Foraminifera of the Antarctic Ocean

Table $\frac{2}{\lambda}$ (Continued)

\begin{tabular}{|c|c|c|c|c|c|c|c|c|c|c|c|}
\hline Station & 4 & 5 & 10 & $3^{\prime}$ & 6 & 3 & 2 & 7 & 9 & 8 & 1 \\
\hline 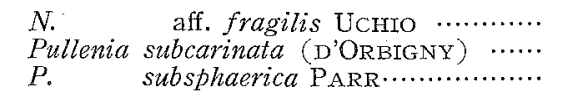 & $\begin{array}{l}\mathrm{vR} \\
\mathrm{vR} \\
-\end{array}$ & $\begin{array}{l}\bar{R} \\
\mathrm{vR}\end{array}$ & $\begin{array}{l}\mathrm{R} \\
\mathrm{VR} \\
\mathrm{VR}\end{array}$ & $\begin{array}{l}\mathrm{vR} \\
\mathrm{R} \\
\mathrm{vR}\end{array}$ & $\overline{\mathrm{R}}$ & $\overline{\mathrm{vR}}$ & $\begin{array}{l}\mathrm{VR} \\
\mathrm{R} \\
\mathrm{VR}\end{array}$ & $\overline{\mathrm{R}}$ & $\begin{array}{l}\mathrm{vR} \\
\mathrm{R} \\
-\end{array}$ & - & $\begin{array}{l}\mathrm{vR} \\
1 \\
\mathrm{vR}\end{array}$ \\
\hline 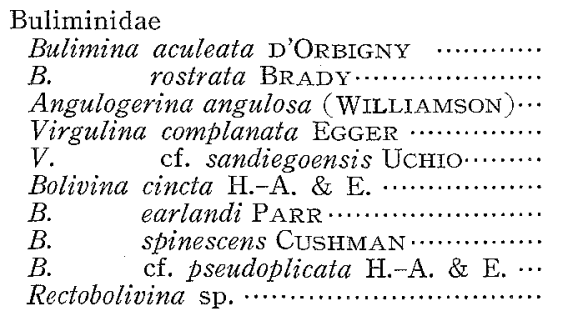 & $\begin{array}{l}\frac{\mathrm{R}}{47} \\
\mathrm{R} \\
\mathrm{R} \\
\mathrm{I} \\
- \\
-\end{array}$ & $\begin{array}{l}\frac{\mathrm{R}}{-} \\
37 \\
- \\
\mathrm{vR} \\
\mathrm{vR} \\
- \\
- \\
-\end{array}$ & $\begin{array}{c}\frac{7}{9} \\
\mathrm{R} \\
\mathrm{R} \\
\mathrm{R} \\
- \\
- \\
-\end{array}$ & $\begin{array}{l}\frac{R}{24} \\
24 \\
\mathrm{VR} \\
\mathrm{R} \\
\overline{\mathrm{R}} \\
\frac{-}{\mathrm{R}}\end{array}$ & $\begin{array}{l}\mathrm{R} \\
\overline{39} \\
- \\
- \\
- \\
- \\
- \\
\mathrm{vR} \\
-\end{array}$ & $\begin{array}{l}\frac{2}{4} \\
42 \\
- \\
- \\
- \\
- \\
- \\
-\end{array}$ & $\begin{array}{l}\frac{2}{42} \\
- \\
- \\
\mathrm{vR} \\
- \\
- \\
-\end{array}$ & $\begin{array}{l}- \\
\overline{9} \\
- \\
- \\
- \\
- \\
- \\
-\end{array}$ & $\begin{array}{l}33 \\
4 \\
1 \\
- \\
\text { R } \\
\mathrm{R} \\
-\end{array}$ & $\begin{array}{l}44 \\
- \\
- \\
- \\
- \\
- \\
-\end{array}$ & $\begin{array}{l}1 \\
\mathrm{vR} \\
\mathrm{R} \\
\mathrm{vR} \\
\mathrm{vR} \\
-\mathrm{R} \\
- \\
-\end{array}$ \\
\hline $\begin{array}{l}\text { Robertinidae } \\
\text { Robertinoides charlottensis (CUSHMAN) } \cdots \\
R . \quad \text { subcylindricus (BRADY) } \cdots \\
\text { Pseudobulimina chapmani (H.-A. \& E.) } \cdots\end{array}$ & $\begin{array}{l}\mathrm{vR} \\
-\end{array}$ & $\overline{-}$ & $\begin{array}{l}\mathrm{vR} \\
-\end{array}$ & $\begin{array}{l}- \\
\overline{\mathrm{VR}}\end{array}$ & $\overrightarrow{\mathrm{L}}$ & - & $\begin{array}{l}\overline{V R} \\
\mathrm{VR}\end{array}$ & $\frac{-}{3}$ & $\underline{\mathrm{vR}}$ & $\overline{-}$ & $\overline{-}$ \\
\hline $\begin{array}{l}\text { Spirillinidae } \\
\text { Spirillina tricarinata WIESNER } \\
\text { S. plana WIESNER ….............. } \\
\text { S. denticulogranulata pulchra PARR } \\
\text { Turrispirillina depressa PARR ................. } \\
\text { Patellina corrugata WILLIAMSON ......... }\end{array}$ & $\begin{array}{l}- \\
\overline{-} \\
\bar{R}\end{array}$ & $\begin{array}{l}\mathrm{vR} \\
\frac{-}{-} \\
\mathrm{vR}\end{array}$ & $\begin{array}{l}-- \\
- \\
-\end{array}$ & $\frac{\mathrm{R}}{-}$ & $\begin{array}{l}- \\
\overline{-} \\
\mathrm{VR} \\
\mathrm{VR}\end{array}$ & $\begin{array}{l}- \\
- \\
-\end{array}$ & $\begin{array}{l}- \\
\overline{\mathrm{R}} \\
-\end{array}$ & $\begin{array}{l}\overline{\mathrm{R}} \\
\mathrm{vR} \\
\overline{\mathrm{vR}}\end{array}$ & $\begin{array}{l}- \\
- \\
-\end{array}$ & $\begin{array}{l}- \\
- \\
- \\
-\end{array}$ & $\begin{array}{l}- \\
- \\
-\end{array}$ \\
\hline 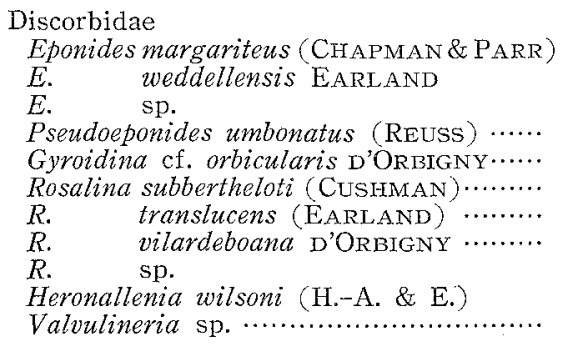 & $\begin{array}{l}\mathrm{R} \\
11 \\
\mathrm{R} \\
- \\
- \\
- \\
- \\
- \\
-\end{array}$ & $\begin{array}{l}\mathrm{vR} \\
2 \\
-\mathrm{vR} \\
\mathrm{vR} \\
\mathrm{vR} \\
\mathrm{vR} \\
\mathrm{vR} \\
- \\
\mathrm{vR} \\
-\end{array}$ & $\begin{array}{l}3 \\
41 \\
\mathrm{R} \\
- \\
- \\
- \\
- \\
- \\
-\end{array}$ & $\begin{array}{l}\mathrm{R} \\
37 \\
\mathrm{R} \\
\overline{\mathrm{vR}} \\
- \\
- \\
- \\
- \\
\mathrm{vR}\end{array}$ & $\begin{array}{l}\overline{2} \\
\overline{\mathrm{vR}} \\
\overline{\mathrm{vR}} \\
\mathrm{vR} \\
\mathrm{vR} \\
\mathrm{vR} \\
\mathrm{vR} \\
-\end{array}$ & $\begin{array}{l}\overline{3} \\
- \\
\overline{\mathrm{vR}} \\
- \\
- \\
- \\
- \\
- \\
-\end{array}$ & $\begin{array}{l}\overline{2} \\
\overline{-} \\
2 \\
- \\
- \\
- \\
\mathrm{VR} \\
-\end{array}$ & $\begin{array}{l}- \\
- \\
- \\
- \\
- \\
- \\
- \\
- \\
\mathrm{VR}\end{array}$ & $\begin{array}{c}6 \\
28 \\
\mathrm{R} \\
\mathrm{R} \\
-- \\
- \\
- \\
- \\
5\end{array}$ & $\begin{array}{l}\overline{22} \\
- \\
- \\
- \\
- \\
- \\
- \\
-\end{array}$ & $\begin{array}{c}3 \\
60 \\
\text { vR } \\
2 \\
\mathrm{R} \\
- \\
- \\
- \\
- \\
- \\
\mathrm{R}\end{array}$ \\
\hline 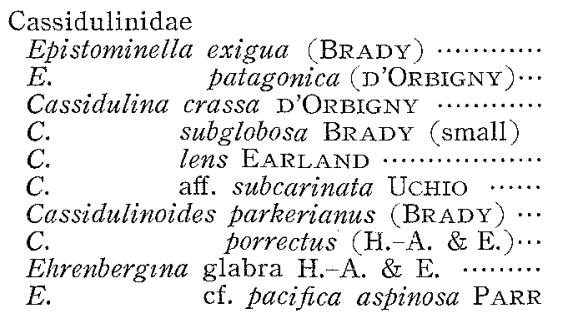 & $\begin{array}{l}\frac{15}{-3} \\
\mathrm{R} \\
\frac{\mathrm{R}}{-} \\
\frac{\mathrm{R}}{-}\end{array}$ & $\begin{array}{l}24 \\
\overline{\mathrm{vR}} \\
6 \\
\frac{\mathrm{vR}}{} \\
-\mathrm{vR} \\
18 \\
-\end{array}$ & $\begin{array}{l}\frac{15}{3} \\
\mathrm{R} \\
\frac{\mathrm{R}}{\mathrm{R}} \\
\overline{\mathrm{VR}} \\
\overline{\mathrm{R}} \\
\end{array}$ & $\begin{array}{l}19 \\
- \\
\overline{3} \\
\overline{\mathrm{vR}} \\
\overline{-} \\
\overline{\mathrm{R}}\end{array}$ & $\begin{array}{l}\frac{13}{5} \\
- \\
- \\
\frac{\mathrm{R}}{-} \\
-\overline{24} \\
-\end{array}$ & $\begin{array}{c}27 \\
\mathrm{R} \\
13 \\
- \\
\mathrm{R} \\
\mathrm{vR} \\
\mathrm{vR} \\
1 \\
-\end{array}$ & $\begin{array}{c}13 \\
\bar{R} \\
17 \\
\mathrm{VR} \\
\mathrm{VR} \\
\mathrm{VR} \\
- \\
4 \\
-\end{array}$ & $\begin{array}{l}\frac{\mathrm{R}}{-} \\
\overline{3} \\
- \\
- \\
\overline{\mathrm{R}} \\
26 \\
--\end{array}$ & $\begin{array}{l}4 \\
- \\
\frac{2}{3} \\
- \\
-\end{array}$ & $\begin{array}{l}\overline{4} \\
- \\
- \\
- \\
- \\
- \\
-\end{array}$ & $\begin{array}{c}\frac{21}{-1} \\
1 \\
1 \\
- \\
-\bar{R} \\
\overline{\mathrm{R}} \\
\mathrm{vR}\end{array}$ \\
\hline $\begin{array}{l}\text { Chilostomellidae } \\
\text { Seabrookia earlandi WRIGHT } \cdots . . . . . . . . . .\end{array}$ & 一 & 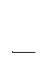 & $\mathrm{vR}$ & $-\cdots$ & - & - & - & - & $\mathrm{VR}$ & - & - \\
\hline $\begin{array}{l}\text { Anomalinidae } \\
\begin{array}{ll}\text { Cibicides grossepunctatus EARLAND } & \text {....... } \\
\text { C. } & \text { lobatulus (WALKER \& JACOB) } \\
\text { C. } & \text { refulgens (MONTFORT) …...... } \\
\text { C. } & \text { sp. } \\
\text { Rupertia stabilis WALLICH .................... }\end{array}\end{array}$ & $\begin{array}{l}\overline{\mathrm{R}} \\
\mathrm{R} \\
\overline{-}\end{array}$ & $\begin{array}{l}\mathrm{R} \\
4 \\
2 \\
\mathrm{R} \\
-\end{array}$ & $\begin{array}{l}\bar{R} \\
\mathrm{R} \\
-\end{array}$ & $\begin{array}{l}\mathrm{vR} \\
\mathrm{R} \\
\mathrm{R} \\
- \\
-\end{array}$ & $\begin{array}{c}\mathrm{R} \\
8 \\
4 \\
-- \\
-\end{array}$ & $\begin{array}{l}2 \\
\mathrm{R} \\
3 \\
- \\
-\end{array}$ & $\begin{array}{l}6 \\
3 \\
2 \\
- \\
-\end{array}$ & $\begin{array}{c}\mathrm{R} \\
3 \\
14 \\
\mathrm{R}\end{array}$ & $\frac{\overline{\mathrm{R}}}{\overline{\mathrm{R}}}$ & $\begin{array}{l}- \\
- \\
-\end{array}$ & $\frac{\mathrm{R}}{-}$ \\
\hline
\end{tabular}




\section{Takayasu Uchio}

EARLAND 1922). The same is true in the Arctic waters where CuSHMAN (op. cit.), PHLEGER (op. cit.) and LOEBLICH \& TAPPAN (op. cit.) found no specimens of Uvigerina, with the single exception that NoRVANG (op. cit., p. 36) found Uvigerina peregrina CUSHMAN along the west and south coast of Iceland. The rare occurrences of many species of arenaceous Foraminifera and Lagena and its related genera (Oolina, Fissurina and Parafissurina) should also be noted. Cushman (1948, p. 44) mentioned the absence of Robulus, Lenticulina and Marginulina in his extensive Arctic material, and PHLEGER (op. cit.) did not find these genera from the Canadian and Greenland Arctic. In the present material Robulus and Marginulina occur rarely. It appears, from a quantitative point of view, that the Lagenidae are not well-developed in the Arctic and Antarctic waters.

The benthonic Foraminifera assemblages consist of at least 37 species which occur in significant abundance at several stations. Twenty species, besides these, have rare (less than 1\%) but rather consistent occurrences. The dominant species are as follows:

\section{Angulogerina angulosa (WILLIAMSON) \\ Bulimina aculeata D'ORBIGNY \\ Eponides weddellensis EARLAND \\ Epistominella exigua (BRADY) \\ Ehrenbergina glabra HERON-ALLEN \& EARLAND \\ Cassidulina subglobosa BRADY (small form)}

The analysis of eleven samples is insufficient to make any definite conclusions even in such a small area. However, the assemblages are so relatively consistent that the results are believed to be reliable. The data and interpretations presented may be modified by the study of additional material, but it is believed that the major features of the distributions are essentially correct.

Depth Boundaries of Benthonic Foraminifera: There appear to be three depth assemblages in the material studied with boundaries at approximately $850 \mathrm{~m}$ and $2,000 \mathrm{~m}$. As mentioned previously, no samples were available shallower than $350 \mathrm{~m}$. There may be additional faunal boundaries, but they are not clear because of the insufficient number of samples and perhaps of faunal mixing. The following species appear to be generally indicative of water depths less than $c a .850 \mathrm{~m}$ :

\footnotetext{
Psammosphaera fusca ScHultze

Hyperammina sp. cf. H. cylindrica PARR

Ammolagena clavata (JONES \& PARKER)

? Alveolophragmium jeffreysii (WILLIAMSON)
} 
? Alveolophragmium wiesneri (PARR)

? Reophax scorpiurus MONTFORT

? Textularia antarctica PARR

? T. earlandi PARKER

Eggerella superba (EARLAND)

Botellina (?) goesii EARLAND

B. (?) tasmanica PARR

Trochammina grisea EARLAND

? $T$. squamata adaperta RHUMBLER

T. antarctica PARR

All Miliolidae (except Pyrgo murrhina) and Ophthalmidiidae

Perhaps all Lagena and related genera

Dentalina communis D'ORBIGNY

D. ittai LOEBLICH \& TAPPAN

Vaginulina subelegans PARR

All Robulus and Darbyella

Glandulina antarctica PARR

Astronoinon antarcticum PARR

A. plana (WIESNER)

Nonion sp. aff. N. zaandamae (VoORTHUYSEN)

Pseudobulimina chapmani (HERON-ALLEN \& EARLAND)

All Spirillinidae

Rosalina subbertheloti (CUSHMAN)

$R$. vilardeboana D'ORBIGNY

$R$. translucens (EARLAND)

Heronallenia wilsoni (HERON-AlLEN \& EARtand)

Cassidulinoides parkerianus (BRADY)

Ehrenbergina glabra HERON-ALLEN \& EARLAND

Pullenia subsphaerica PARR

Cibicides refulgens (MONTFORT)

C. lobatulus (WALKER \& JACOB)

C. grossepunctatus EARLAND

The following species have a significant change in frequency at $c a .850 \mathrm{~m}$ :

Bulimina aculeata D'ORBIGNY

Angulogerina angulosa (WILLIAMSON)

Eponides weddellensis EARLAND

Epistominella exigua (BRADY)

The following species appear to be restricted to depths deeper than $c a .2,000 \mathrm{~m}$ :

Haplophragmoides bradyi (ROBERTSON)

Bulimina rostrata BRADY 
Nonion sp. cf. N. colligerum (CHAPMAN \& PARR)

Pyrulina extensa (CushmaN)

The following species have a significant change in frequency at $c a .2,000 \mathrm{~m}$ :

Bulimina aculeata D'ORBIGNY

Eponides weddellensis EARLAND

Epistominella exigua (BRADY)

Benthonic Foraminifera Assemblages: Three assemblages are recognized based on the depth boundaries discussed above.

Assemblage 1 (depth 350-850 m) may be called the Angulogerina angulosaEpistominella exigua-Ehrenbergina glabra assemblage. Important species are:

Psammosphaera fusca, Ammolagena clavata

Alveolophragmium jeffreysii, Trochammina antarctica, Astrononion antarcticum, A. plana, Bulimina aculeata, Pseudobulimina chapmani, Patellina corrugata,

Heronallenia wilsoni, Eponides weddellensis,

Cassidulina crassa, C. subglobosa, C. sp. cf. C. subcarinata,

Pullenia subcarinata, P. subsphaerica, Cibicides lobatulus,

$C$. refulgens, and $C$. grossepunctatus.

Assemblage 2 (depths $850-2,000 \mathrm{~m}$ ) may be called the Bulimina aculeata assemblage. Eponides weddellensis is also dominant. Only 24 benthonic specimens were found in all the material (ca. 7.1 grams of mud) at Station 8. Therefore, the percentage distribution of species in this sample is not so reliable as it is in other samples. There is no doubt, however, that the Bulimina aculeata is most dominant at Station 8. More samples are necessary to establish this assemblage.

Assemblage 3 (deeper than 2,000 m) may be called the Eponides weddellensisEpistominella exigua assemblage. These two species are also dominant in the other assemblages, but Eponides weddellensis has the highest frequency in this one. Haplophragmoides bradyi, Nonion sp. cf. N. colligera, Pyrulina extensa and Bulimina rostrata are characteristic of this assemblage.

It is desirable to compare these assemblages with those in other parts of Antarctica. No quantitative analysis of Antarctic Foraminifera has been published previously, although there are many taxonomic papers. There are just as many taxonomic papers dealing with Arctic waters as Antarctic, but a few based on quantitative works done by STSCHEDRINA (1947) and PHLEGER (op. cit.). PhlEGER (op. cit., p. 88) says, "Considerable faunal mixing is obvious in the samples and it is suggested that this is caused by ice-rafting. Faunal 
Benthonic Foraminifera of the Antarctic Ocean

mixing and thus absence of clearcut depth zonation may be a characteristic of Arctic areas, especially those areas in which there is considerable shallow water around an intricate coastline. Ice transportation of sedimentary material, including Foraminifera from near shore zones and later deposition in deeper waters is probably one of the main sedimentary processes in Arctic and Antarctic regions." The present writer believes that PHLEGER's statement is correct as a general principle. It is not easy, however, to say which species are displaced or to what extent, since almost nothing is known about the distributions of living benthonic Foraminifera in Arctic and Antarctic regions. PHLEGER's finding of 13 species (no depth and quantitative distributions) in the Arctic region and the writer's finding of 11 species in the Antarctic region, mentioned above, constitute all that is known.

The following species in Assemblage 3 may be displaced from shallower waters :

Pelosinella bicaudata PARR

Involutina sp. A

Textularia earlandi PARKER

Trochammina squamata adaperta RHUMBLER

Cibicides lobatulus (WALKER \& JACOB)

C. grossepunctatus EARLAND

Nonion sp. aff. N. zaandamae (VOORTHUYSEN)

Most of miliolids and ophthalmiids may be displaced from depths much shallower than $350 \mathrm{~m}$. Specimens of Cornuspiroides lacunosa (BRADY), Rupertia stabilis WALLICH and Ehrenbergina sp. cf. E. pacifica aspinosa PARR are discolored and may be fossils. As a whole, these probably displaced specimens are negligible in the area studied, very likely due to the simple submarine topography and deep continental shelf whose outer edge lies at depths of $c a$. 500-600 m (Shoji \& SATo, op. cit., p. 41). Another factor which makes the depth zonation of the benthonic assemblages obscure and causes apparently mixed or displaced fauna is the presence of pre-modern sediment on the sea floor (Uchio, 1960\% This is discussed later in this paper.

\section{INTERPRETATION OF THE BENTHONIC ASSEMBLAGES}

Since the Foraminifera assemblages mentioned above are thanatocoenoses and not biocoenoses, there may be no direct relationship between them and the hydrographic conditions. It is desirable, however, to correlate the assemblages and physical factors as much as possible to get a better understanding of the former. Two important facts should be borne in mind when interpreting thanatocoenoses in the Antarctic waters. One is that most specimens of all 
species are transported toward deeper waters to a greater or less extent after the death of the organisms and before their burial in sediment. This is demonstrated clearly by UcHIO (1960, text-figs. 2--9) off the coast of Southern California. The other is that the bottom water of the Indian, Pacific and South Atlantic Oceans is formed in the Antarctic Ocean, particularly in the Weddell Sea, which makes the interpretation of benthonic faunas in the Antarctic Ocean more difficult than in other waters of the world.

In routine oceanographic observations no bottom water is collected, but bottom conditions (temperature, salinity, etc.) are inferred by extrapolating the data obtained from the water column (see Fig. 2A). The extrapolation method cannot be applied to the Antarctic region where the Antarctic Bottom Water is formed in winter (see Fig. 2B). According to SvERDRUP et al. (1946, p. 611), the water on the Antarctic continental shelf, the salinity of which may be increased up to $34.62 \%$ or slightly higher by freezing, has the greatest density (temperature $c a .-0.4^{\circ} \mathrm{C}$ : salinity $c a .34 .66 \%$; density, $\sigma_{t} c a .27 .89$ ) and sinks down along the continental slope; while sinking it is mixed with the warmer and more saline circumpolar water of somewhat lower density (temperature $c a .0 .5^{\circ} \mathrm{C}$; salinity ca. 34.68\% ; density, $\sigma_{t} c a .27 .84$ ), and bottom water is formed which has a slightly greater density (temperature $c a .-0.4^{\circ} \mathrm{C}$; salinity ca. 34.66\% ; density, $\sigma_{t} c a .27 .86$ ) than the circumpolar water. The formation of ice on the Antarctic continental shelves is of primary importance to the Antarctic Bottom Water. Thus it is apparent that the oceanographic conditions at a point on the bottom ( $A_{0}$ in Fig. $2 B$ ) may be quite different from these of the water column at the same depth $\left(A_{0}^{\prime}, A_{1}\right.$ and $A_{2}$ in Fig. $\left.2 B\right)$. Therefore, in winter the sea floor at all the stations occupied may be covered by the Antarctic Bottom Water, while in summer, when the present samples were collected, the situation is quite different. According to the summer hydrographic data (Ishino, Morita \& SAOTOME, 1958) during the First Japanese Antarctic Research Expedition 1956-57, the bottom at Station 4 was covered by the Antarctic Surface Water or by the uppermost part of the Antarctic Circumpolar Water; Stations 5, 10, 3',6,3,2, 7 and 9 by the Antarctic Circumpolar Water; and Station 1 by the Antarctic Bottom Water. The sea floor at Station 8 might have been covered by the Antarctic Bottom Water but the benthonic Foraminifera assemblage suggests that the station was covered by the Antarctic Circumpolar Water. Thus, Assemblage 1 appears to represent the Antarctic Circumpolar Water and in part the lowermost part of the Antarctic Surface Water; Assemblage 2 the lower part of the Antarctic Circumpolar Water; and Assemblage 3 the Antarctic Bottom Water. It appears that the summer hydrographic conditions clearly affect benthonic Foraminifera assemblages but the winter ones do not.

The above interpretation is a simplification of the real situation since the 
bottom sediments at these stations are not necessarily Recent sediments now being deposited there. The writer, in another paper on planktonic Foraminifera of this area, has found that the sediment at Station 2 is $c a .5,500$ years old, and the sediments at the other stations are also probably pre-modern. If so, it may or may not be correct to correlate pre-modern benthonic assemblages

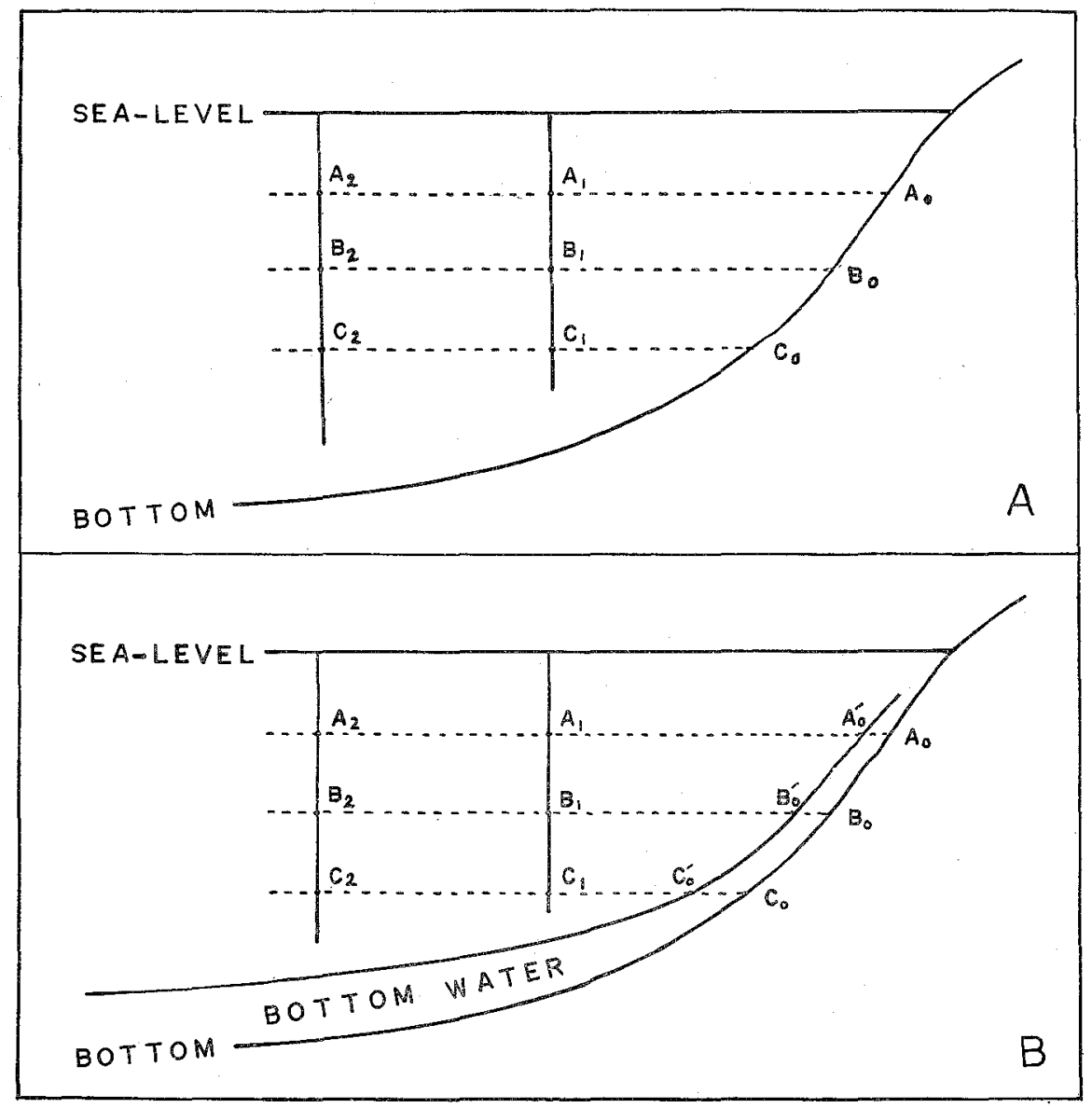

Fig. 2. Schematic diagram showing how to infer bottom conditions by extraporating the data obtained from the water column.

$$
\begin{aligned}
& \text { A : where the extraporation method can be applied. } \\
& \text { B : where the extraporation method cannot be applied. }
\end{aligned}
$$

with Recent oceanographic conditions. To get a final solution of such a complicated problem it is necessary to collect more sediment samples and to make a $\mathrm{C}^{14}$ dating and Foraminifera analysis of each one. Furthermore, the distribution of living benthonic Foraminifera must be studied as a basis for the interpretation of the distribution of empty tests of Foraminifera. 


\section{Takayasu Úchio}

\section{DISCUSSION}

The result obtained by SHoJI \& SATO (op. cit.) from the same samples is primarily concerned with the grain size analysis of the sediment. (1) According to them the cumulative frequency curve of grain size is bimodal or trimodal at most of the stations, and $\sigma_{\phi}$ value, which is indicative of the sorting of the sediment, is generally large. From these facts as well as the fact that the gravels (and/or pebbles) are angular or subangular and variable in lithology, they consider that the gravels are ice-rafted but that the sands and muds are transported by ocean currents. The writer, however, thinks that all of the gravels and a large part of the sand and mud are ice-rafted and that some are relicts of pre-modern sediment, as mentioned above.

(2) SHoJI and SATO show that the weight percentage of Foraminifera (planktonic plus benthonic) content in the sediment samples is generally $1-3 \%$, which is considered to indicate the Foraminifera production in this area. They also say that Foraminifera content is higher, ca. 10-20\%, on Gunners Bank and they attribute this partly to current transportation but primarily to the higher productivity of the water due to topographic upwelling on the bank. The present writer has examined their statements and would like to comment as follows:

First, the Foraminifera content in the sediment does not necessarily indicate the productivity of the water at present, because the Foraminifera at Station 2 are $c a .5,500$ years old as indicated by $\mathrm{C}^{11}$ dating. However, the productivity of the sea water at present may not be essentially different from that of 5,500 years ago.

Secondly, the Foraminifera content of the sediment on the bank is not always much higher than on the continential shelf and slope, as is shown in Fig. 3A. Assuming higher productivity on the bank than on the shelf or slope, it still cannot be attributed to topographic upwelling as they believe. It is well known that high concentrations of phosphate, and hence of plankton, occur in the South Atlantic around Antarctica, as shown by SvERDRup et al. (1946, p. 787, fig. 217 ; p. 786, fig. 216) and these phenomena are attributed to convection. Sverdrup et al. (op. cit., p. 790) discuss convection as follows: "In regions where there are marked seasonal changes in temperature between winter and summer, the changes are of great importance not only because of the effect of temperature directly upon the rate of metabolism but also because of its indirect effect in renewal of nutrients to the surface layers. This method of renewal is characteristic of fresh water lakes, but is also very important in the sea, especially in the higher latitudes. The phenomenon is, of course, dependent upon the surface waters cooling to a point where their density becomes sufficient to cause them to sink and be replaced by upward movement 
of lighter and incidentally nutrient-rich waters from below." Around Antarctica, particularly in the Weddell Sea, the water on the continental shelf freezes in winter and sinks along the bottom. This water is replaced by an upward movement of the Antarctic Deep Water, which is then called the Antarctic Circumpolar Water. This is schematically shown by SvERDRUP et al. (op. cit., p. 620, fig. 164). Therefore, a high Foraminifera content of sediment is expected regardless of submarine topography.

The apparently higher Foraminifera content (?) on the bank than on the shelf or slope must be explained in some way other than the productivity of sea water. The washing out of fine detritus (silt and clay) from sediment, causing concentration of Foraminifera tests on the bank is one possibility. On the

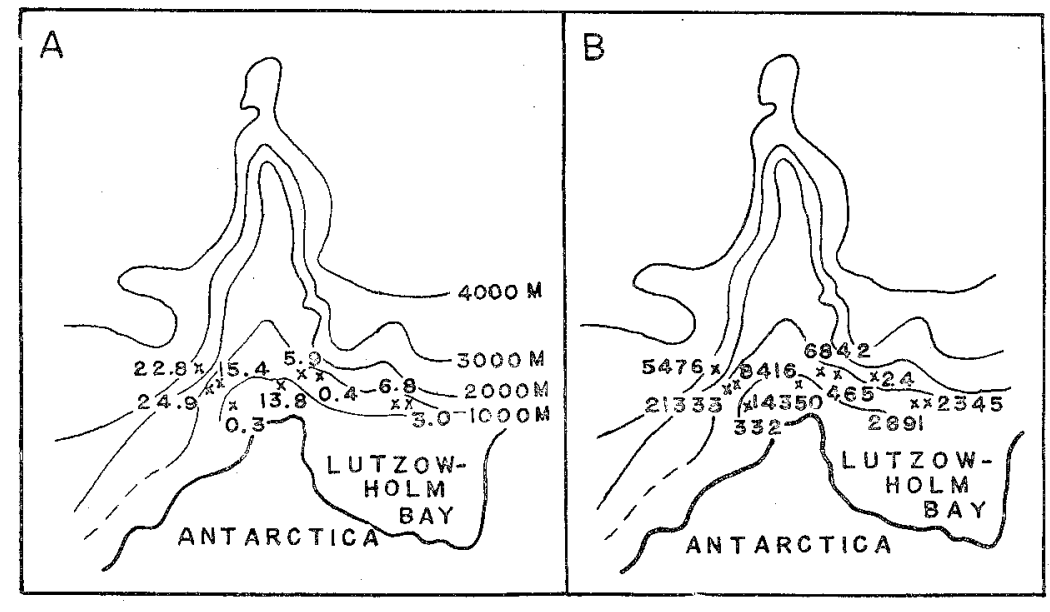

Fig. $3 \mathrm{~A}$

Fig. 3B

Fig. 3A. Weight percentages of Foraminifera content in sediment (Sand fraction only)

Fig. 3 B. Numbers of Foraminifera tests in one gram of dried sediment.

other hand, the Foraminifera content of sediment is not definitely higher on the bank than on the shelf or slope. The Foraminifera content, grain size distribution, etc., of sediment are expected to be very changeable or patchy, and cannot be determined accurately in glacial regions. Therefore, the distributions of Foraminifera content, as well as sediment type, based on a few stations in such regions may be very misleading. ShoJI and SATo do not say how they estimated the Foraminifera content of the sediment. Perhaps they did this by weighing the Foraminifera floated by carbon tetrachloride or by calculating $\mathrm{CaCO}_{3}$ content dissolved by adding acid to the sediment. The writer counted the Foraminifera tests in the sediment microscopically (see Table 2, Fig. 3B). Relative abundances of the Foraminifera content based on 


\section{Takayasu Uchio}

the number of Foraminifera tests in one gram of dried sediment at these stations are similar to those based on the weight of the Foraminifera, as is to be expected (see Table 3, also compare Fig. $3 \mathrm{~A}$ with Fig. 3B).

Table 3. Relative abundance of Foraminifera content based on number of Foraminifera tests in one gram of dried sediment and on the weight percentage of Foraminifera Content. The latter is calculated from data given by SHOJI \& SATO. Stations are arranged in order of increasing depths.

\begin{tabular}{c|c|c|c|c|c|c|c|c|c|c|c}
\hline Station & 4 & 5 & 10 & $3^{\prime}$ & 6 & 3 & 2 & 7 & 9 & 8 & 1 \\
\hline Foram. No./Foram. No. at Sta. 4 & 1 & 43 & 7 & 8 & 21 & 25 & 64 & 1.4 & 9 & 0.07 & 17 \\
\hline Foram. Weight/Foram. Weight at Sta. 4 & 1 & 46 & 10 & - & 19 & 51 & 83 & 1.3 & 23 & - & 76 \\
\hline
\end{tabular}

(3) The depth of the continental shelf is much deeper than those in other areas. SHOJI and SATo interpret this in two ways: (1) crustal subsidence due to the very thick continental ice cap, (2) glacial erosion. They prefer the first interpretation because of the presence of extremely well sorted fine sand (excluding gravels and Foraminifera tests), which they believe to be relict of ancient beach sand on Gunners Bank. Their belief in the presence of ancient sediment on the bank is supported by the writer's finding of $c a$. 5,500 years old sediment at Station 2. However, the benthonic Foraminifera assemblages at Station 2 and at the other stations show that this sediment cannot be beach sand unless the sediment predates the assemblage.

\section{REFERENCES}

BRADY, H.B. 1884. Report on the Foraminifera dredged by H.M.S. "Challenger" during the years 18731876. Rept. Voy. Challenger, Zoology, vol. 9, 1 vol. text 814 pp., 1 vol. pls. 1-115.

Chapman, F. \& PARR, W.J. 1937. Foraminifera: Australasian Antarctic Exped., 1911-1914. Sci. Rept. ser. C, Zoology and Botany, vol. 1, pt. 2, pp. 1-190, pls. 7-10.

CUSHMAN, J.A. 1910-1917. A Monograph of the Foraminifera of the North Pacific Ocean. U.S. Nat. Mus., Bull. 71, pts. 1-6. 1918-1931. The Foraminifera of the Atlantic Ocean. ibid., Bull. 104, pts. 1-8. 1945. Foraminifera of the United States Antarctic Service Expedition 1939-1941. Proc. America: Philosoph. Soc., vol. 89 , no. 1, pp. 285-288, a plate.

pls. 1-8.

EARland, A. 1933. Foraminifera, Part 2, South Gerogia. Discovery Repts., vol. 7, pp. 27-138, pls. 1-7. -1934. Foraminifera, Part 3, The Falklands Sector of the Antarctic (excluding South Georgia). Discovery Repts., vol. 10, pp. 1-208, pls. 1-10.

1936. Foraminifera, Part 4, Additional Records from Material obtained by the S. Y. "Scotia". Discovery Repts., vol. 13, pp. 1-76, pls. 1-2A (with a report on some crystalline components of the Weddell Sea deposits by F. A. BANNISTER with chemical analyses by M. H. HEY).

Flint, J.M. 1899. Recent Foraminifera. Ann. Rept. U.S. Nat. Mus. for 1897, pp. 249_349, pls. 1_80.

Heron-Allen, E. \& Earland, A. 1922. Protozoa, Part 2, Foraminifera. British Antarctic ("Terra Nova”) Expedition, 1910, Natural History Rept., Zoology, vol. 6, no. 2, pp. 25-268, 8 plates. 


\section{Benthonic Foraminifera of the Antarctic Ocean}

Heron-Allen, E. \& Earland, A. 1932. Foraminifera, Part 1, The ice-free area of the Falkland Island and adjacent seas: Discovery Repts., vol. 4, pp. 291-460, pls. 6-17.

HöGLund, H. 1947. Foraminifera in the Gullmar Fjord and the Skagerak. Zool. Bidrag Uppsala, vol. 26, pp. 1-328, pls. 1-32.

Ishino, M., Morita, Y. \& SAotome, Y. 1958. Note on the oceanographical surveys in the Indian Ocean and the Southern Ocean (in Japanese with English abstract). Jour. Tokyo Univ. Fisheries, (Special edition), vol. 1, no. 3, pp. 103-240.

Loeblich, A.R. \& TAPPAN, H. 1953. Studies of Arctic Foraminifera. Smithsonian Miscellaneous Collections, vol. 121, no. 7 , pp. 1-150, pls. 1-24.

NAKASEKO, K. 1959. On Radiolaria from sediments in the sea near Antarctica, Part 1, On superfamily Liosphaericae (Radiolaria) from sediments in the sea near Antarctica. Biological Results of the Japanese Antarctic Research Expedition, no. 2, 13 pp., 3 pls.; (Spec. Publ. Seto Marine Biological Laboratory).

NinNo, H., 1958. On the bottom deposits of the sea around Cape Cook, Prins Harald Coast, Antarctic region (in Japanese with English abstract). Jour. Tokyo Univ. Fisheries, (Special edition), vol. 1, no. 3 , pp. $250-254,2$ pls.

PARR, W.J. 1950. Foraminifera. B.A.N.Z. Antarctic Research Exped. 1929-31, Rept., ser. B (Zoology \& Botany), vol. 5, pt. 6, pp. 235-392, pls. 3-15.

PEARCEy, F.G. 1914. Foraminifera of the Scottish National Antarctic Expedition. Trans. Roy. Soc. Edinburg, vol. 49, pt. 4, pp. 991-1044, pls. 1-2.

PhLEGER, F.B 1952. Foraminifera distribution in some sediment samples from the Canadian and Greenland Arctic. Contr. Cushman Found. Foram. Res., vol. 3, pt. 2, pp. 80-89, pls. 13-14.

SholI, D. \& Sato, T. 1959. On the bottom sediment dredged by the Second Japanese Antarctic Exploration-Especially on the grain size analysis (in Japanese with English abstract). Antarctic Record, no. 7, pp. 39-49.

Stschedrina, Z. G. 1947. On the distribution of the Foraminifera in the Greenland Sea: Repts. Acad. Sciences USSR, vol. 55, no. 9, pp. 871-874 (in Russian).

SVERDrup, H.U., JoIINSON, M.W. \& FLEMING, R.H. 1946. The Oceans, their physics, chemistry and general biology. Prentice Hall, New York, 1087 pp.

UCHIO, T. 1959. Foraminifera assemblages in bottom sediments and in plankton-net samples in the Antarctic Ocean (abstract) (in Japanese). Jour. Geol. Soc. Japan, vol. 65, no. 766, p. 443.

1960. Ecology of living benthonic Foraminifera from the San Diego, California, area. Cushman Found. Foram. Res., Spec. Publ. No. 5, 72 pp., 10 plates.

1960. Planktonic Foraminifera of the Antarctic Ocean. Biological Results of the Japanese Antarctic Research Expedition, no. 11, pp. 1-9, a plate (Spec. Publ. Seto Marine Biological Laboratory).

WaLton, W.R., 1952, Techniques for recognition of living Foraminifera. Contr. Cushman Found. Foram. Res., vol. 3, pt. 2, pp. 56-60.

Warthin, A.S. 1934. Foraminifera from the Ross Sea. American Museum Novitates, vol. 71, p. 1-4, figs. $1-5$.

WIESNER, H. 1931. Die Foraminiferen der Deutschen Südpolar-Expedition 1901-1903. Deutsche SüdpolarExped., vol. 20 (Zoologie, vol. 12), pp. 53-165, pls. 1-24. 


\section{PLATE I}

Figs. 1-3. The Angulogerina angulosa-Epistominella exigua-Ehrenbergina glabra

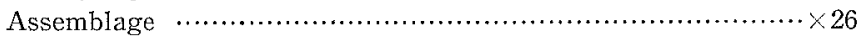

Fig. 1............ Station 5 (depth $490 \mathrm{~m}$ )

Fig. 2 and $3 \cdots$ Station 6 (depth $630 \mathrm{~m}$ )

Fig. 4. The Bulimina aculeata Assemblage ................................ 26 Station 9 (depth $870 \mathrm{~m}$ )

Fig. 5. The Eponides weddellensis-Epistominella exigua Assemblage $. . . \ldots \ldots \times 26$ Station 1 (depth $2480 \mathrm{~m}$ ) 
Plate I
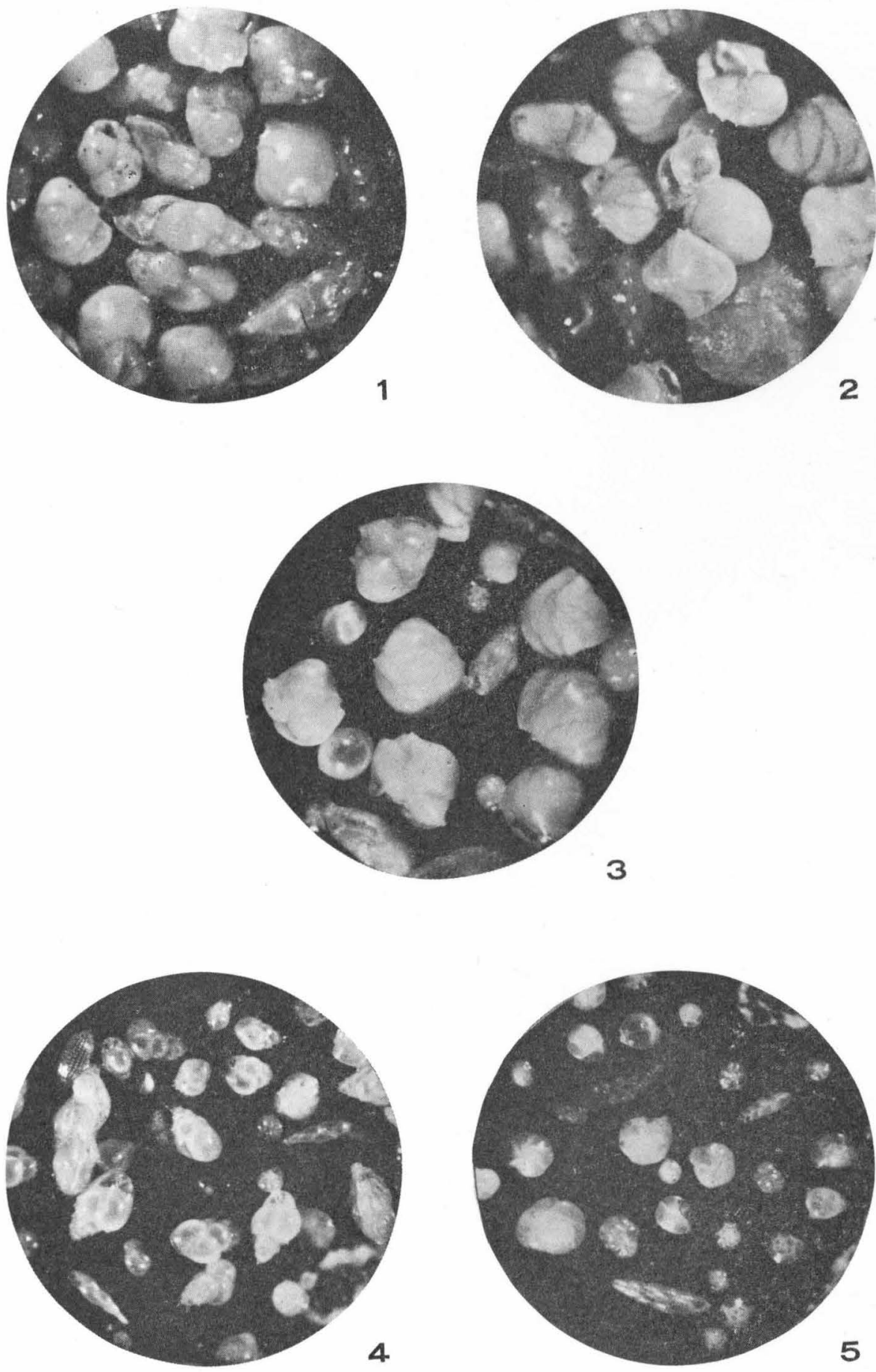


\section{BIOLOGICAL RESULTS \\ $\mathrm{OF}$ \\ THE JAPANESE ANTARCTIC RESEARCH EXPEDITION}

1. Tanita, Senji: Sponges. 1959.

$¥ 50$

2. NAKASEKo, Kojiro: On Superfamily Liosphaericae (Radiolaria) from sediments in the sea near Antarctica (On Radiolaria from sediments in the sea near Antarctica. Part 1). 1959.

$¥ 150$

3. Hirano, Minoru: Notes on some algae from the Antarctic collected by the Japanese Antarctic Research Expedition. 1959.

$¥ 150$

4. HataI, Kotora: A new rhynchonellid (Brachiopoda) from Antarctica. 1959. 50

5. Токіока, Takasi : Amaroucium erythraeum Michaelsen, a compound ascidian from the Cape Province. 1959.

$¥ 50$

6. YosII, Riozo: Collembolan fauna of the Cape Province, with special reference to the genus Seira Lubbock. 1959.

$¥ 150$

7. Gamô, Sigeo: On a cumacean Crustacea (Diastylis corniculatus Hale) obtained by the Second Japanese Antarctic Research Expedition (1957-58). 1959 . $¥ 50$

8. Utinomi, Huzio: Pycnogonida of the Japanese Antarctic Research Expeditions 1956-1958. 1959.

$¥ 100$

9. Matsubara, Kiyomatsu and Iwai, Tamotsu: Fishes. 1959 . $¥ 50$

10. Tanaka, Otohiko: Pelagic Copepoda. 1960 . 700

11. Uchro, Takayasu: Planktonic Foraminifera of the Antarctic Ocean. 1960. $¥ 100$

12. Uchro, Takayasu: Benthonic Foraminifera of the Antarctic Ocean. 1960. $¥ 150$ 